\title{
Quantifying the uncertainties in a fault stability analysis of the Val d'Agri oilfield
}

\author{
Daniele Rossi ${ }^{1} \cdot$ Anna Scotti ${ }^{1} \cdot$ Luigi Vadacca $^{1}$
}

Received: 12 June 2019 / Accepted: 2 April 2020

(c) Springer-Verlag GmbH Germany, part of Springer Nature 2020

\begin{abstract}
Induced seismicity as an effect of injection of fluids in a geological basin is a widely observed phenomenon which is nowadays under public scrutiny. In most cases, the numerical simulation and interpretation of these phenomena requires models with a high degree of realism. In turn, this requires accounting for complex interactions between the fluid and solid components, as well as a detailed description of geometry and a high degree of heterogeneity in the material properties. Also, issues related to the uncertainty of parameters, to an incorrect modeling of sub-scale or multiscale effects and to a limited knowledge of initial conditions are unavoidable and can be detrimental to the reliability of the results. In this work, a statistical analysis including uncertainty quantification and sensitivity analysis on variances has been applied as a post-processing to data coming from a set of numerical simulations of a real world setting (the Val D'Agri oilfield), with the aim of studying the stability of a fault that is known to have experienced a good amount of microseismicity during the modeled period. The uncertainty quantification targeted the effects of fault surface local orientation and pore pressure fluctuations, as well as variability in the friction coefficient of the fault. The analysis of variances focused on the effects of varying the permeability of the fault damage zones (the area enveloping the faults) and the geometrical orientation of the fault as well. The model shows that the zone where the microseismicity has been measured is included in a wider region of moderate instability, which is higher the lower the permeability of the fault damage zone. From the model results the fault seems to be far from a critical state, but the analysis offers, nevertheless, some useful information on the relationship of slip tendency with geometrical and flow quantities in the system, and suggests some improvements in the dynamical model assumptions and settings.
\end{abstract}

Keywords Uncertainty quantification $\cdot$ Sensitivity analysis $\cdot$ Poromechanics $\cdot$ Fault reactivation

Mathematics Subject Classification 62P12

Daniele Rossi

daniele.rossi@polimi.it

Extended author information available on the last page of the article 


\section{Introduction}

The numerical modeling of geophysical systems has received a lot of interest since the first appearance of modern computing science itself. Given the advances both in computing power and numerical techniques, the number of possible applications of numerical simulation has steadily risen, and many of them are relevant for common human activities (most notably related with weather prediction, engineering and risk assessment). Nevertheless, the modeling of such systems comes with the unavoidable issue of the reliability of the results, subject as they are to both model and data limitations.

The need to assess how reliable the model results are at given conditions implies some sort of probabilistic interpretation of the assumptions and data. Various techniques have been developed over the years, and they are usually grouped under the general terms of uncertainty quantification and sensitivity analysis. Uncertainty Quantification (UQ) aims at finding the amount of uncertainty in an output given the variability of a set of data or assumptions, while Sensitivity Analysis (SA) searches for a quantification of both the absolute and relative importance of the various parameter uncertainties on the results. Geophysical and environmental applied studies, with the great amount of inherently uncertain parameter values and strongly non-linear behavior, are a perfect area of application for these techniques. Uncertainty quantification and sensitivity analysis have been extensively used to study atmosphere and ocean (Kalra et al. 2017; Aleksankina et al. 2018), hydrology (Roy et al. 2018), nuclear waste disposal (Saltelli and Tarantola 2002), or basin and crust studies (Maina and Guadagnini 2018; Colombo et al. 2018; Formaggia et al. 2013).

The importance of an uncertainty evaluation is even greater when dealing with systems subject to strong nonlinearities, extreme conditions or abrupt/discontinuous change of state past some threshold. This is the case for severe weather (Molini et al. 2009), climate change (Peng et al. 2002), floods (Hall and Solomatine 2008), and fault reactivation (Vidal-Gilbert et al. 2009). The study of fault reactivation in geological systems, among the others, meets strong interest from the scientific community, industry and policy makers. Particular attention is put on cases in which reactivation can be caused by injection of fluids into the subsurface related to human activities, such as oil production and $\mathrm{CO}_{2}$ sequestration.

The material composing the shallower part of the earth's crust is brittle and rock layers contain fractures. If those fractures at some point experience tangential displacements, they are called faults. Such faults, subject to tectonic forces, can either slide at a regular speed (creeping motion) or accumulate the stresses in form of elastic energy and suddenly release it, dissipating part of the energy locally because of friction, and transmitting the rest in form of seismic waves and slip along the fault plane (Turcotte and Shubert 2014).

When fluid is injected through a well it causes an increase of pore pressure, which, reducing the effective normal stress, leads to a weakening of the fault and can cause its sliding (induced seismicity). The effect has been known since 1960s, but with the recent increase of injection operation from industrial explorations both observation of the phenomenon and discussions of possible hazards have since multiplied (Moeck et al. 2009; Worum et al. 2004; Bao et al. 2014; Pereira et al. 2014; Ellsworth 2013). 
Recently, microseismicity induced by wastewater injection has been observed and analyzed in depth, thanks to an unusually large amount of data available, from the events occurred in Val d'Agri in early 2010s. (Stabile et al. 2014; Improta et al. 2015; Buttinelli et al. 2016). Val d'Agri is situated in the Southern Apennines (Italy), and it hosts the largest on-shore oilfield in Europe. It is an extensional basin characterized by a complex fault system (Buttinelli et al. 2016).

In 2015, it included 25 oil wells with total production rates around 90 barrels/day. The wastewater associated with oil production has been re-injected in an unproductive well (CM2) since June 2006, with maximum injection rates between $6 \times 10^{4}$ and $8 \times 10^{4} \mathrm{~m}^{3} /$ month. The injection well is located between two oppositely dipping normal faults (Improta et al. 2015; Buttinelli et al. 2016) (see Fig. 1). During the injection period, the area was subject to various measurements: a dense network managed by INGV(Istituto Nazionale Geofisica e Vulcanologia) active until the first half of June 2006, and networked monitoring both from the ENI (Ente Nazionale Idrocarburi, an Italian energy company) and the regular INGV network. From the analysis of seismic
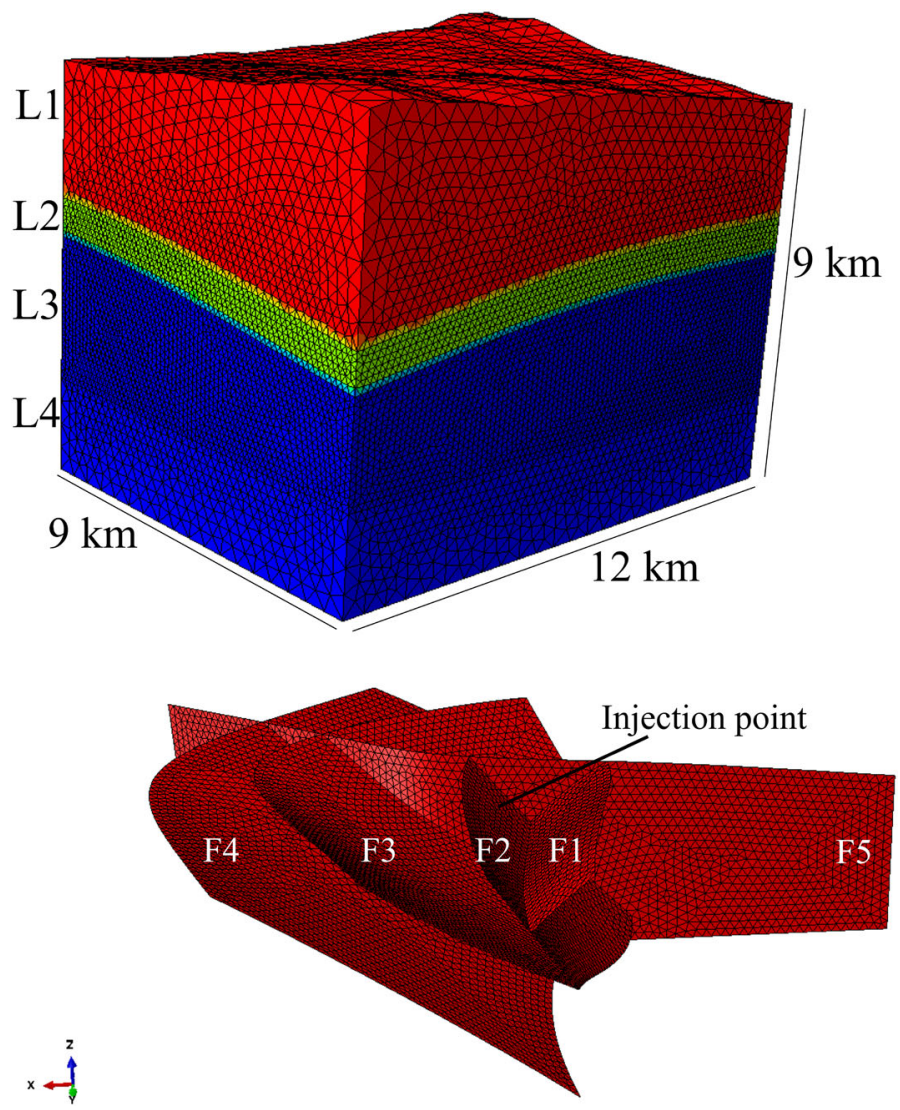

Fig. 1 The model domain (on the top); The fault system (on the bottom). F1 is the one considered in the statistical analysis 
data, in Improta et al. (2015), the authors found a strict correlation between microseismicity (earthquakes with magnitude $M_{w} \leq 2.2$ ) and wastewater injection rates. In addition, the quick onset and migration of seismicity suggested a rapid propagation of pore pressure perturbations within a high permeability damage zone (Improta et al. 2015).

In this paper, uncertainty quantification tools are adapted and applied to a numerical study of the aforementioned case.

The paper is structured as follows: Sect. 2 describes the governing equations and their numerical discretization, Sect. 3 lists the two main methods used in the statistical analysis, Sect. 4 describes the result of the analysis, and Sect. 5 draws the conclusions.

\section{Numerical simulation}

The results subject to statistical analysis are provided by geophysical simulations run using the software (ABAQUS 2013), a commercial finite element suite.

\subsection{Governing equations}

Let $\Omega$ be a three-dimensional domain containing $N_{f}$ embedded surfaces $\Gamma_{i}$, with $i=1, \ldots N_{f}$ representing the faults. We also define $\Gamma=\bigcup_{i=1}^{N} \Gamma_{i}$ as the union of such surfaces. Across each $\Gamma_{i}$ material properties and variables can be discontinuous, so, when necessary, we will denote with a superscript ${ }^{+}$and $^{-}{ }^{-}$its vaules on the sides of the surface. The governing equations for the problem of poroelasticity in the presence of faults are two conservation equations and a condition on the displacement across $\Gamma$. The conservation of fluid mass reads:

$$
\frac{\partial m}{\partial t}+\nabla \cdot \mathbf{w}=\rho_{f} f
$$

where $m$ is the fluid mass, $f$ is a volumetric source term, $\rho_{f}$ the fluid density and $\mathbf{w}=\rho_{f} \mathbf{v}$, with the fluid velocity $\mathbf{v}$ determined by the Darcy equation. Note that the fluid mass $m$ changes as the result of changes in porosity, i.e. due to mechanical deformations, and, to a lesser extent, due to compressibility. We can write its time derivative as:

$$
\frac{\partial m}{\partial t}=\frac{\partial(\nabla \cdot \mathbf{u})}{\partial t} \rho_{f}+\frac{1}{M} \frac{\partial p_{f}}{\partial t}
$$

where $M$ accounts for the compressibility of the fluid and the solid grains, the divergence of the displacement $\mathbf{u}$ describes the volume change associated with the deformation of the solid skeleton and $p_{f}$ is the fluid pressure. Denoting with $\mathbf{k}$ the permeability tensor, with $\mu_{f}$ the dynamic viscosity of the fluid and with $\mathbf{g}$ the gravitational acceleration vector, the Darcy equation reads:

$$
\mathbf{v}=\frac{\mathbf{k}}{\mu_{f}}\left(\nabla p_{f}-\rho_{f} \mathbf{g}\right) .
$$


Throughout this work, the permeability is considered to be isotropic, so that $\mathbf{k}=k \boldsymbol{\delta}$, where $\delta$ is the unit tensor.

From the point of view of fluid flow the fault core, geometrically represented by $\Gamma$, is modeled as an interface with finite permeability $\kappa_{c}$ (ABAQUS 2013). Considering that the fault core is extremely thin and has a permeabilty several orders of magnitude lower than its surrounding, we consider that no noticeable amount of fluid can move tangentially inside the core itself. Thus, we neglect the flow inside the fault surfaces $\Gamma$ but allow the flow across them. This, in turn, can cause a jump in pressure between the two sides of $\Gamma$. The fault core is surrounded by an envelope of damaged rocks of normal permeability where the fluid can move normally. For similar reasons, denoting with $\mathbf{n}_{\Gamma}$ the normal to the fault and pointing towards the + side, we have that:

$$
\mathbf{v}^{+} \cdot \mathbf{n}_{\Gamma}=\mathbf{v}^{-} \cdot \mathbf{n}_{\Gamma},
$$

i.e. the normal flux across the fault is continuous since we have set its storativity to zero. Thus, we have the interface conditions for the fluid problem:

$$
\left\{\begin{array}{l}
\mathbf{v}^{+} \cdot \mathbf{n}_{\Gamma}=-\frac{\kappa_{c}}{\mu_{f}}\left(p_{f}^{+}-p_{f}^{-}\right) \\
\mathbf{v}^{-} \cdot \mathbf{n}_{\Gamma}=-\frac{\kappa_{c}}{\mu_{f}}\left(p_{f}^{+}-p_{f}^{-}\right) .
\end{array}\right.
$$

The second equation to describe a poroelastic system consists in the mechanical equilibrium equation:

$$
\nabla \cdot \boldsymbol{\sigma}+\rho_{b} \mathbf{g}=\mathbf{0} .
$$

Here, $\boldsymbol{\sigma}$ is the Cauchy total stress tensor, and $\rho_{b}$ is the bulk density, defined as $\rho_{b}=$ $\phi \rho_{f}+(1-\phi) \rho_{s}$, where $\rho_{s}$ is the density of the solid and $\phi$ is the porosity.

For a porous medium fully saturated with water, the relation between stress and effective stress $\boldsymbol{\sigma}^{\prime}$ is expressed by the Terzaghi effective stress principle:

$$
\boldsymbol{\sigma}^{\prime}=\boldsymbol{\sigma}-p_{f} \boldsymbol{\delta}
$$

If the effective stress tensor is expressed in a reference system where it becomes diagonal, it is usually useful to figure it as an ellipsoid where the three eigenvalues represent the semi-axes, and are numbered in decreasing order of their absolute value as $\sigma_{1}, \sigma_{2}, \sigma_{3}$ (Wangen 2019). For the experiments in question, the tectonic stress is such that $\sigma_{1}$ is expected to align with the vertical direction, $\sigma_{2}$ with the $y$ axis and $\sigma_{3}$ with the $x$ axis.

We recall that, in a poroelastic medium, displacement is linked to the effective stress according to a constitutive equation that can be written as:

$$
\boldsymbol{\sigma}^{\prime}=\mathcal{C}^{d r}: \boldsymbol{\epsilon}(\mathbf{u})
$$


where $\epsilon$ is the deformation and $\mathcal{C}^{d r}$ is the fourth order modulus tensor whose component, for the isotropic elastic response case, are expressed as:

$$
\mathcal{C}_{i j k l}^{d r}=G\left(\delta_{i k} \delta_{j l}+\delta_{i l} \delta_{j k}\right)+(K-2 G / 3) \delta_{i j} \delta_{k l},
$$

where $G$ and $K$ are the bulk and shear modulus, and are functions of the Young modulus $E$ and Poisson ratio $v$ :

$$
\begin{aligned}
G & =\frac{E}{2(1+v)} \\
K & =\frac{E}{3(1-2 v)}
\end{aligned}
$$

Values for these parameters as used in our model are displayed in Table 1.

If the stress tangential to the fault becomes large enough, the fault can slip. This relative displacement is a vector $\mathbf{d}$ along the fault plane $\Gamma$ :

$$
\mathbf{d}=\mathbf{u}^{+}-\mathbf{u}^{-} \text {on } \Gamma
$$

with the additional requirement that $\mathbf{d}$ lies on the surface $\Gamma$, so that the fault can slide without opening.

The condition for fault slip comes from Amonton's Law, which states that the maximum tangential stress is proportional to the effective normal stress on the surface,

$$
\tau=\mu_{\Gamma} \sigma_{n}^{\prime}
$$

Here, $\sigma_{n}^{\prime}=\left(\boldsymbol{\sigma}^{\prime} \mathbf{n}_{\Gamma}\right) \cdot \mathbf{n}_{\Gamma}$, with $\mathbf{n}_{\Gamma}$ the normal to the fault surface, stands for the normal component of the effective normal traction, $\tau$ is the shear stress modulus while the shear traction vector can be computed as $\boldsymbol{\tau}=\boldsymbol{\sigma} \mathbf{n}_{\Gamma}-\sigma_{n} \mathbf{n}_{\Gamma} \cdot \mu_{\Gamma}$ is the sliding friction coefficient, whose typical values range from 0.6 to 0.8 , but can be less of 0.4 in the presence of clay minerals (Collettini et al. 2009). We can then define the Slip Tendency as

$$
S T=\frac{\tau}{\sigma_{n}^{\prime}} .
$$

Table 1 Hydromechanical properties of the layers. Rock density $\rho_{s}$, Young modulus $E$, Poisson ratio $v$, Bulk modulus $K_{f}$, porosity $\phi$ and permeability $k$

\begin{tabular}{lllllll}
\hline Layer & $\rho_{s}\left(\mathrm{~kg} / \mathrm{m}^{3}\right)$ & $E(\mathrm{GPa})$ & $v$ & $K_{f}(\mathrm{GPa})$ & $\phi$ & $k\left(\mathrm{~m}^{2}\right)$ \\
\hline L1 & 2520 & 32.16 & 0.30 & 2.2 & 0.1 & $10^{-15}$ \\
L2 & 2610 & 49.16 & 0.28 & 2.2 & 0.08 & $10^{-19}$ \\
L3 & 2830 & 84.35 & 0.26 & 2.2 & 0.05 & $10^{-14}$ \\
L4 & 2830 & 84.35 & 0.26 & 2.2 & 0.049 & $10^{-16}$ \\
\hline
\end{tabular}


If $S T<\mu_{\Gamma}$ no slip occurs and the stress state is stable. If not, the strength of the fault is overcome and slip may start to propagate along the fault plane. We can thus partition the fault surface $\Gamma$ into two regions: a locked part $\Gamma_{L}$ and a slipping part $\Gamma_{S}$. This partitioning evolves during the simulation, i.e. a point which is initially stuck can fail and start slipping if the tangential stress exceeds the maximum value, and conversely a slipping point can stop sliding if the conditions change. On the locked part of the fault we will impose a given value of the displacement jump $(\overline{\mathbf{d}})$, whereas on the slipping portion we impose the limit tangential traction according to Amonton's law.

The above equations can be combined obtaining a system for the unknowns: displacement $\mathbf{u}$ and fluid pressure $p_{f}$. The complete system thus reads:

$$
\begin{cases}\nabla \cdot\left(\boldsymbol{\sigma}^{\prime}(\boldsymbol{u})+p_{f} \boldsymbol{\delta}\right)+\rho_{b} \boldsymbol{g}=\mathbf{0} & \text { in } \Omega \\ \frac{1}{M} \frac{\partial p_{f}}{\partial t}+\frac{\partial \epsilon_{v}(\boldsymbol{u})}{\partial t}-\rho_{f} \cdot\left(\frac{\boldsymbol{k}}{\mu_{f}} \nabla p_{f}-\rho_{f} \boldsymbol{g}\right)=\rho_{f} f & \text { in } \Omega \\ \mathbf{u}^{+}-\mathbf{u}^{-}=\overline{\mathbf{d}} & \text { on } \Gamma_{L} \\ \boldsymbol{\tau}=-\mu_{\Gamma} \sigma_{n}^{\prime} \frac{\mathbf{d}}{\|\dot{\mathbf{d}}\|} & \text { on } \Gamma_{S} \\ -\frac{\boldsymbol{k}}{\mu_{f}} \nabla p_{f}^{ \pm} \cdot \mathbf{n}_{\Gamma}=\frac{\kappa_{c}}{\mu_{f}}\left(p_{f}^{+}-p_{f}^{-}\right) & \text {on } \Gamma\end{cases}
$$

where $\dot{\mathbf{d}}$ indicates the time derivative of the displacement $\mathbf{d}$.

Note that the traction on $\Gamma_{S}$ depends on the effective normal pressure, the friction coefficient and its direction such that it opposes the slip velocity. Moreover, we remark that due to the frictional contact the problem is strongly nonlinear and needs to be solved by means of a suitable iterative method. In particular, the algorithm implemented in Abaqus starts with a guess for the slip vector $\mathbf{d}$, then, after the solution of the elasticity problem compares the tangential stress with the limit value and determines whether the point has failed, then iterates these steps pointwise until convergence.

\subsection{Experimental setting and input parameters}

The structure of the geophysical system (layers and faults) is deduced from published data (Buttinelli et al. 2016) and corresponds to the part of the Val d'Agri oilfield affected by induced seismicity. The domain is a volume of $9 \times 12 \times 9 \mathrm{~km}$, with the top boundary following the topographic surface (see Fig. 1). The volume is occupied by 4 layers that differ in rock density, Young modulus, Poisson ratio, porosity and permeability. The corresponding values are reported in Table 1 . The pores are assumed to be fully saturated with water with $\rho_{f}=10^{3} \mathrm{~kg} / \mathrm{m}^{3}$.

The volume contains 5 embedded surfaces representing the faults, as shown in Fig. 1. The faults and their proximities have properties that differ from the rest of the medium. We distinguish between the faults' core, represented by frictional contacts and a low permeability (see Sec. 2.1), and the surrounding damage zone modeled by means of an envelope of mesh elements around the contacts where it is possible to set permeability values $k_{d}$ that differ from the original surrounding rock. 
Table 2 Variations of permeability of the damage zone $\left(k_{d}\right)$ and fault cores $\left(k_{c}\right)$

\begin{tabular}{llll}
\hline Experiment & $k_{d}\left(\mathrm{~m}^{2}\right)$ & $\kappa_{c}\left(\mathrm{~m}^{2}\right)$ & $\mu_{\Gamma}$ \\
\hline A1 & $10^{-13}$ & $10^{-19}$ & 0.6 \\
A2 & $10^{-14}$ & $10^{-19}$ & 0.6 \\
A3 & $10^{-15}$ & $10^{-19}$ & 0.6 \\
\hline
\end{tabular}

$\mu_{S}$ is the sliding friction coefficient

The computational grid is a tetrahedral mesh which honors the geometry of the faults, i.e. the faults are approximated by faces of the grid elements. In particular we have employed a mesh of approximately $3 \times 10^{6}$ tetrahedra.

The mesh nodes on $\Gamma$ are split, this means that the nodes along the fault surfaces are duplicated, new node-IDs are assigned to the new nodes, and the mesh connectivity is updated. In this way, each fault is characterized by two surfaces that are geometrically coincident but distinct from the numerical point of view: one surface belongs to the hanging-wall block and the second one to the foot-wall block (Vadacca et al. 2018). The nodes on the perimeter of the fault are instead merged, as it is assumed that no crack propagation phenomena occur.

As for the boundary conditions, atmospheric pressure is imposed at the top boundary, together with a zero-flux condition on the other boundaries.

The time integration of the model has been conducted using a three stage approach, with two sequential preparation steps and a simulation step.

1. In the first preparation step the model was subjected to gravitational loading and to an initial uniaxial stress field.

2. In the second preparation step a velocity of $\pm 3 \mathrm{~mm} / \mathrm{y}$ has been applied in the $x$ direction, normal to the boundaries orthogonal to the $x$-axis simulating the extensional tectonic regime active in the region.

3. In the simulation step, the injection from the well bottom was added and the model was run for almost 8.5 years. The injection rate is shown in Fig. 3. It approximately reproduces the injection rate reported by Improta et al. (2015).

Three experiments have been conducted with the same methodology, varying the permeability of the faults damage zones. The permeability ranges from the same permeability as the surrounding host rock (setup A3, with $k_{d}=10^{-15} \mathrm{~m}^{2}$ ) to a value 2 orders of magnitude higher (A1). The data for the three experiments are listed in Table 2. The permeability of the fault core and the friction coefficient are kept the same for all the simulations (Vadacca et al. 2019).

\section{The statistical analysis tools}

In this section we present two groups of methods developed with two goals in mind: (a) accounting for the unpredictability in the stress tensor and local errors in fault geometry and (b) evaluating the effects of the permeability of the fault damage zone on the stability of the fault. This is possible since the total stress is continuous across faults and can thus be interpolated, for instance, at different fault locations. In order to 
answer to (a), a UQ method has been chosen, where the effect of normal deviation from the mean values is analyzed. The strategy chosen to solve (b) has been a sensitivity analysis based on the analysis of variances.

The dynamical model, due to its sheer cost compared to the available computational resources, was unsuitable to be run even for a moderate number of times on the same case study. This means that the development of the statistical tools for the analysis has focussed on the idea of applying it as post-processing to a very limited numer of geomechanical simulations (one in the case of the uncertainty quantification, three in the case of the sensitivity analysis) performed with reference values. At the same time, this choice operates a selection on the parameters suitable to the analysis. Variation on parameters as the ones shown in Table 1, for example, cannot be considered without running the whole model many times.

The quantity of interest for these experiments is the Slip Tendency (ST) [see Eq. (13) and Sec. 2.1]. The parameters used in our statistical analysis are: the dip (zenithal) and strike (azimuthal) angles, the pore pressure $p_{f}$, the permeability of the faults damage zone and the uncertainty in the fault friction coefficient. More specific considerations about the quantification and treatment of the uncertainty in relation with the various parameters are made in Sec. 4.

\subsection{The uncertainty quantification method}

For the UQ method, we have taken into account the uncertainty related to three different quantities:

- The geometric orientation of the fault surface, that can be expressed as variations in the zenithal (dip) and azimuthal (strike) angle of the normal to the surface.

- The effects of fluctuations in the pore pressure, which directly influence fault stability. From the phenomenological point of view, this is like assuming the presence of small scale variations that can locally influence the outcome of the flow, whose effects however are not strong enough to change the shape of the stress tensor.

- The uncertainty on the friction coefficient $\mu_{\Gamma}$, whose value determines the strength of the fault (Sibson 1985).

The error distributions are assumed to be normal for both angles as well as for $p_{f}$. The fluctuations included in the statistical analysis are going to be small, the ST changes smoothly with the angles (cfr. Wangen 2019 ch. 3 ) and the variation in the $p_{f}$ alone cannot change the ST pattern [i.e. the direction of the principal stresses, see (Morris and Ferrill 2009)]. Thus, given also the relatively large number of points to be analyzed, the normal distributions are sampled at regularly spaced intervals. Denoting by $e_{\xi}$ the standard deviation of a normally distributed random variable $\xi$ with zero mean, the interval $\left[-3 e_{\xi}, 3 e_{\xi}\right]$ is divided into $N_{\xi}$ intervals of equal length $\delta \xi$. A pair of values is assigned to each interval. The first is the weight $w_{j}^{\xi}$ of the $j$ th interval, defined as:

$$
w_{j}^{\xi}=\frac{1}{\sqrt{2 \pi} e_{\xi}} \int_{\tilde{\xi}_{j}}^{\tilde{\xi}_{j+1}} \exp \left(\frac{-\xi^{2}}{2 e_{\xi}^{2}}\right) \mathrm{d} \xi=\Phi_{0, e_{\xi}}\left(\tilde{\xi}_{j+1}\right)-\Phi_{0, e_{\xi}}\left(\tilde{\xi}_{j}\right)
$$


where $\tilde{\xi}_{j}, \tilde{\xi}_{j+1}$ are the endpoints of the interval $\left[\tilde{\xi}_{j}, \tilde{\xi}_{j}+\delta \xi\right]$, and $\Phi_{m, e_{\xi}}(\xi)$ is the normal cumulative distribution function:

$$
\Phi_{m_{\xi}, e_{\xi}}(\xi)=\frac{1}{2}\left[1+\operatorname{erf}\left(\frac{\xi-m_{\xi}}{\sqrt{2} e_{\xi}}\right)\right]
$$

where $m_{\xi}$ is the value of expectation of the random function $\xi$, set as 0 in Eq. (15) because it is assumed that the mean is equal to the deterministic value.

The second is the representative value $\xi_{j}$ of the $j$ th interval, chosen as the value at which the area of the distribution curve between $\left[\tilde{\xi}_{j}, \xi_{j}\right]$ and $\left[\xi_{j}, \tilde{\xi}_{j+1}\right]$ is the same:

$$
\Phi_{0, e_{\xi}}\left(\xi_{j}\right)=\frac{1}{2}\left(\Phi_{0, e_{\xi}}\left(\tilde{\xi}_{j+1}\right)+\Phi_{0, e_{\xi}}\left(\tilde{\xi}_{j}\right)\right)
$$

These definitions make it possible to add two additional points at the extremes of the distribution, for the intervals $[-\infty,-3 e]$ and $[3 e, \infty]$ given the values of $\operatorname{erf}(-\infty)=-1, \operatorname{erf}(+\infty)=1$.

In our uncertainty quantification we consider the fluctuations in the pore pressure, dip and strike angle, denoted exchanging $\xi$ with $p, d$ and $s$ respectively. From the point of view of the numerical implementation, the fault normal is computed for each triangular element composing the fault surface. Values and weights are stored in arrays for a normalized standard deviation, so that the real values can be obtained by a simple rescaling.

Details on how the parameters are chosen can be found in the next Sect. (4).

Since the friction coefficient $\mu_{\Gamma}$, which should be compared to the slip tendency to assess stability, can be also affected by uncertainty, we define a new quantity called Probability of Slip (PS), which is obtained weighting each ST evaluation with a weighting function $\mathcal{W}_{\mu_{\Gamma}}(\mathrm{ST})$, so that the PS is defined as:

$$
\mathrm{PS}=\sum_{i=0}^{N_{d}} \sum_{j=0}^{N_{s}} \sum_{k=0}^{N_{p}} w_{i}^{d} w_{j}^{s} w_{k}^{p} \mathcal{W}_{\mu_{\Gamma}}\left(\mathrm{ST}_{i, j, k}\right)
$$

where $N_{d}, N_{s}$ and $N_{p}$ are the number of sample for the dip, strike and pore pressure distributions respectively. If the Heaviside function $\Theta$ were chosen for $\mathcal{W}$ $\left(\mathcal{W}_{\mu_{\Gamma}}(\mathrm{ST})=\Theta\left(\mathrm{ST}-\mu_{\Gamma}\right)\right)$, then the friction coefficient would be considered as a deterministic threshold, i.e. the contribution to the probability of slip would be zero for each evaluation such that $S T \leq \mu_{\Gamma}$. However, if we characterize the uncertainty of $\mu_{\Gamma}$ by its standard deviation $e_{\mu}$ from the expected value we can use the cumulative distribution function as a weighting to compute the probability of slip:

$$
\mathcal{W}_{\mu, e_{\mu}}(\mathrm{ST})=\Phi_{\mu_{\Gamma}, e_{\mu}}(\mathrm{ST})
$$

where $e_{\mu}$ is the standard deviation attributed to the friction coefficient $\mu_{\Gamma}$. 


\subsection{The sensitivity analysis}

The analysis of variances is based on the seminal work of Sobol (1993). In what follows, we will restrict the description for a 3-parameter case, which will be sufficient for our model.

Let's consider a random function $p$, which depends on a set of parameters $x_{i}, i=$ 1,3 . If we consider a parameter interval normalized on a cube $K^{3}$ with sides of unit length, we can write the average value of $p$ on it.

$$
\bar{p}=\int_{K^{3}} p\left(x_{1}, x_{2}, x_{3}\right) \mathrm{d} \mathbf{x}=\int_{0}^{1} \int_{0}^{1} \int_{0}^{1} p\left(x_{1}, x_{2}, x_{3}\right) \mathrm{d} x_{1} \mathrm{~d} x_{2} \mathrm{~d} x_{3}=p_{0}
$$

if we integrate $p$ on the square composed by any two of the three variables and subtract $p_{0}$, we get the function (with $i_{1} \neq i_{2} \neq i_{3}$ ):

$$
p_{i_{1}}\left(x_{i_{1}}\right)=\int_{0}^{1} \int_{0}^{1} p\left(x_{1}, x_{2}, x_{3}\right) \mathrm{d} x_{i_{2}} \mathrm{~d} x_{i_{3}}-p_{0}
$$

whose integral with respect to $x_{i_{1}}$ is zero. This means also that the set of function $p_{i_{1}}$ $(i=1,2,3)$ are orthogonal, in fact for $i \neq j$ :

$$
\int_{K^{3}} p_{i_{1}} p_{i_{2}} \mathrm{~d} \mathbf{x}=0
$$

In a similar way it is possible to define:

$$
p_{i_{1} i_{2}}\left(x_{i_{1}}, x_{i_{2}}\right)=\int_{0}^{1} p\left(x_{1}, x_{2}, x_{3}\right) \mathrm{d} x_{i_{3}}
$$

It is then possible to decompose the original random function $p$ with the series:

$$
p=p_{0}+\sum_{i_{1}=1}^{3} p_{i_{1}}+\sum_{i_{1}=1}^{3} \sum_{i_{2}=i_{1}+1}^{3} p_{i_{1} i_{2}}+p_{123}
$$

or:

$$
p=p_{0}+p_{1}+p_{2}+p_{3}+p_{12}+p_{13}+p_{23}+p_{123}
$$

If $p \in L^{2}$, it can be used to define the centered second order moment (variance):

$$
D^{2}=\int_{K^{3}} p^{2}(x) \mathrm{d} \mathbf{x}-p_{0}^{2}
$$


Squaring Eq. (24), considering also Eq. (22), one gets:

$$
D^{2}=\sum_{i_{1}=1}^{3} D_{i_{1}}^{2}+\sum_{i_{1}=1}^{3} \sum_{i_{2}=i_{1}+1}^{3} D_{i_{1} i_{2}}^{2}+D_{123}^{2}
$$

where:

$$
D_{i_{1}, \ldots, i_{s}}^{2}=\int_{0}^{1} \ldots \int_{0}^{1} p_{i_{1}, \ldots, i_{s}}^{2} \mathrm{~d} x_{i_{1}} \ldots \mathrm{d} x_{i_{s}}
$$

At this point, following Sobol (1993), the sensitivity $S_{i}$ can be obtained from the variances simply by:

$$
S_{i_{1}, \ldots, i_{s}}=D_{i_{1}, \ldots, i_{s}}^{2} / D^{2}
$$

In this work, we apply the analysis of variances to compare the effects of the variations in the fault angles with those of different values of permeability of the fault damage zone.

With this choice of variable Eq.(27) can be rewritten simply as:

$$
D^{2}=D_{k}^{2}+D_{d}^{2}+D_{s}^{2}+D_{k, s}^{2}+D_{k, d}^{2}+D_{k, d, s}^{2},
$$

where the subscripts $k, d$ and $s$ denote permeability, dip and strike respectively, and the various components can be computed using Eqs. (20) and (28).

Since the computations are limited to a post processing of only 3 simulations, to obtain the values of stress for all possible sampled values of permeability we perform an interpolation of the stress tensor among the three given simulation results as follows. As stated before, the three simulations (A1, $A 2$ and $A 3)$ are run with different permeability of the damage zones (for the values of the permeability look at Table 2). For each element of $\boldsymbol{\sigma}$, the interpolation on the $k_{d}$ value is done using a pair of quadratic polynomial (one for each of $k_{d}$ intervals $\left[\log \left(10^{-13}\right), \log \left(10^{-14}\right)\right]$ and $\left.\left[\log \left(10^{-14}\right), \log \left(10^{-15}\right)\right]\right)$, imposing the continuity of the derivative on the central point $\left(k_{d}=\log \left(10^{-14}\right)\right)$.

Also for this analysis, since ST changes smoothly with the angles and we expect small changes in the principal stress orientations for the effects of varying the permeability (Morris and Ferrill 2009), the distribution is considered to be smooth enough to not require a Monte Carlo method. The number of samples is computed at equally spaced points, and are considered of equal probability.

\section{Results and discussion}

The fault system is shown in Fig. 1. The fault F1 is the one where the micro-earthquake swarm was observed (Buttinelli et al. 2016).

The data for the Val D'Agri oil field in our possession for that time are the one published by Improta et al. (2015) and Buttinelli et al. (2016), and are restricted for the 
most part to the seismicity data and the shape and position of the fault, extracted from geophysical data. We do not have access to borehole data that could let us estimate in situ stresses, thus they are deduced from regional data (Montone and Mariucci 2016).

Given the position of the injection point (nearer to the fault F2 than to F1), and the fact that both F1 and F2 show similar orientation with respect to the tensional stress, the appearence of the micro-seismicity on F1 is unexpected, unless assuming some variability in our data. Because of this, in this stage of the work we focused our analysis on the fault F1 in order to find possible sources of local instability.

The triangulated surface of the fault has been used to compute the normals for each of its elements. Given the node splitting on the surface, the interpolation has been made on the two elements nearest to the fault, one from the top and one from the bottom, and for the ST evaluation the higher value of the two sides has been considered (as in Jha and Juanes (2014)).

The geomechanical simulation has been run using Abaqus on an HPC network. It used 32 processes on a single node with Xeon E5-4610 V2-2.3GHz processors and a total of $240 \mathrm{~GB}$ of RAM. The simulations took about $40 \mathrm{~h}$ to complete. After that, the relevant data (stresses, pore pressure) for the elements in contact with the fault F1 have been extracted and the computation of the statistics has been conducted on a laptop with a Python script.

The uncertainty quantification has been carried out for each element composing the fault surface at each timestep. 9 values have been sampled for each uncertain parameter, obtaining a total of 729 evaluations per element per timestep.

Given the limited knowledge of the data, the range of variation of each parameter had to be chosen based on discussions and heuristic considerations. We summarize shortly how they have been chosen.

Angles (strike and dip): The angle is decomposed in its zenithal (dip) and azimuthal (strike) component. Considering that the typical dimension of a surface element side is between 50 and $100 \mathrm{~m}$, a standard deviation of $5^{\circ}$ has been chosen. This translates in a standard deviation between two points of 4-8 $\mathrm{m}$. The deviation is applied as follows: the normal to the surface is converted in spherical coordinates, then the deviations are applied to the angles and the new spherical coordinates are used to compute the 'perturbed' normal.

Pore pressure: The uncertainty in the transport of fluid in the model is considered only for its effect on the final pore pressure. This approximation is expected to be reliable only for small fluctuation, as localized overpressure should influence deformation and stresses. The hydrostatic dependence of pressure makes unpractical to fix a single value for the pressure variation. A relative error $e_{r}=0.02$ has been chosen instead. The fluctuation are extracted from a normal distribution with a standard deviation of $e_{r} p_{f}$ and added to the normal stress.

Permeability of the damage zone: The evaluation of the effect of permeability of the damage zone should have complex effect on transport of fluid and final stresses. In this model an evaluation of the effects has been accomplished doing 3 simulation with different values of damage zone permeability $k_{d}$ and interpolating the value of the stress tensor between them. The range of variation has been chosen in order to include values suggested in other works (Improta et al. 2015), and ranges 
between $10^{-13}$ and $10^{-15} \mathrm{~m}^{2}$. More information on the method used can be found in Sect. 3.2.

Friction: Variation on the friction coefficient $\mu_{\Gamma}$, being the actual threshold for slip initiation, can have a fundamental impact on the final result. The amount of local variation of $\mu_{\Gamma}$ is connected to the knowledge of the local variation in the materials and couldn't be assessed from the data at our disposal. Typical value of friction coefficient for carbonates is commonly assumed to be 0.6 with variations that can decrease up to 0.5 (Scuderi and Collettini 2016). In the model the standard deviation of $\mu_{\Gamma}$ was set to be 0.05 on a basic value of $\mu_{\Gamma}=0.6$. No further considerations are made on the spatial variation of the material, as this would require many dynamic simulations.

Figure 2 shows the average values of ST as well as the PS (see Eq. 18) map for the F1 fault for the experiments A1, A2 and A3. Both quantities are greater the lower is the permeability $k_{f}$. The data are taken from the final timestep. The instability is maximum on the borders of the fault, where the nearby surface of other faults or the domain boundary are limiting fluid motion. In none of the cases the ST exceeds the friction coefficient, and the PS computed with the non-deterministic threshold does not exceed 0.05 . With a higher permeability, the fluid gets transported away from the source more efficiently and the stability is higher.

In Fig. 3, the ST averaged over all the triangles is plotted versus the time of the simulation. It can be seen how the profiles follow the rate of injection, with greatest value for case A3 and the lowest for case A1. In the same figure, the square root of the variances of the ST are reported. We divide them between the variance among point (which covers about $4 / 5$ of the total), and the variance among the set of values of each point. This subdivision is similar to the one used in dispersion inside fluids (see Csanady 1973 p. 84). If $\overline{\text { ST }}$ stands for the local average for the single surface triangle over the UQ set, and $\langle S T\rangle$ stands for the spatial average, then defining the spatial average of the variance on each triangle:

$$
e_{L}^{2}=\left\langle e^{2}\right\rangle=\left\langle\left(\overline{(\mathrm{ST}-\overline{\mathrm{ST}})^{2}}\right)\right\rangle .
$$

and the spatial variance of the triangle averages:

$$
\left.e_{S}^{2}=\sqrt{(\overline{\mathrm{ST}}-\langle\overline{\mathrm{ST}}\rangle)^{2}}\right\rangle
$$

The total variance is $e_{T}^{2}=e_{S}^{2}+e_{L}^{2}$.

The variance $e_{S}^{2}$ is in an inverse relationship with its average value, showing that the increase of pore pressure due to the injection from the well reduces the spatial disparities inside the fault surface, whereas the average local variance $e_{L}^{2}$ is subject to an increase. Comparing the experiments with different permeabilities with each other, it is possible to see that the variations between the $e_{L}$ values are not very pronounced. Conversely, in the $e_{S}$ values there is an indication that, at least in this scenario, to a higher permeability in the damage zone corresponds a higher variablity in the ST and that the increase of pore pressure produced by injection has a stronger impact on the reduction of spatial variabilities the higher the damage zone permeability. 

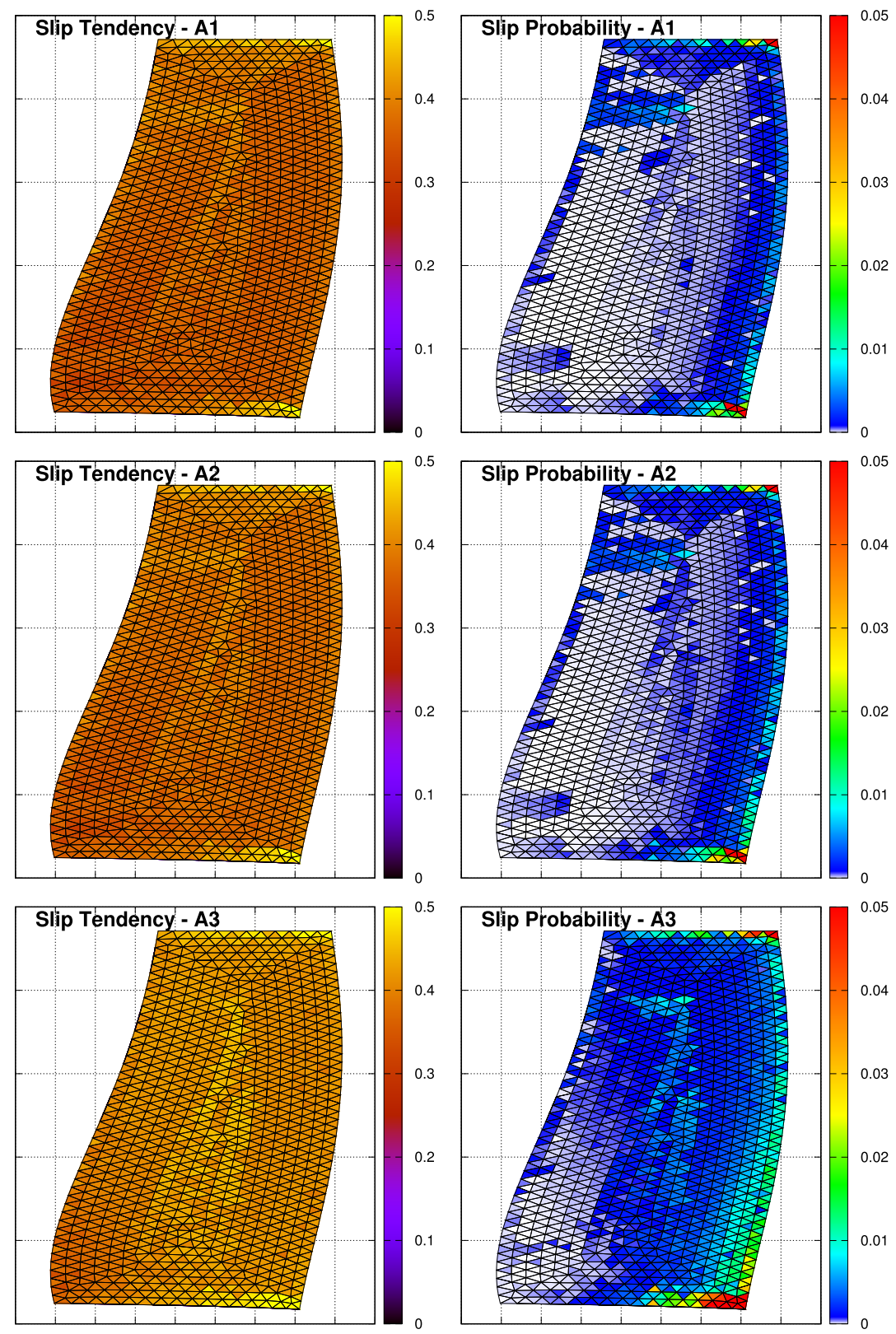

Fig. 2 On the left column the the values of ST are shown for the case A1(top), A2 (middle), A3(bottom). On the right column, the corresponding values of PS. The data refers to the last timestep 

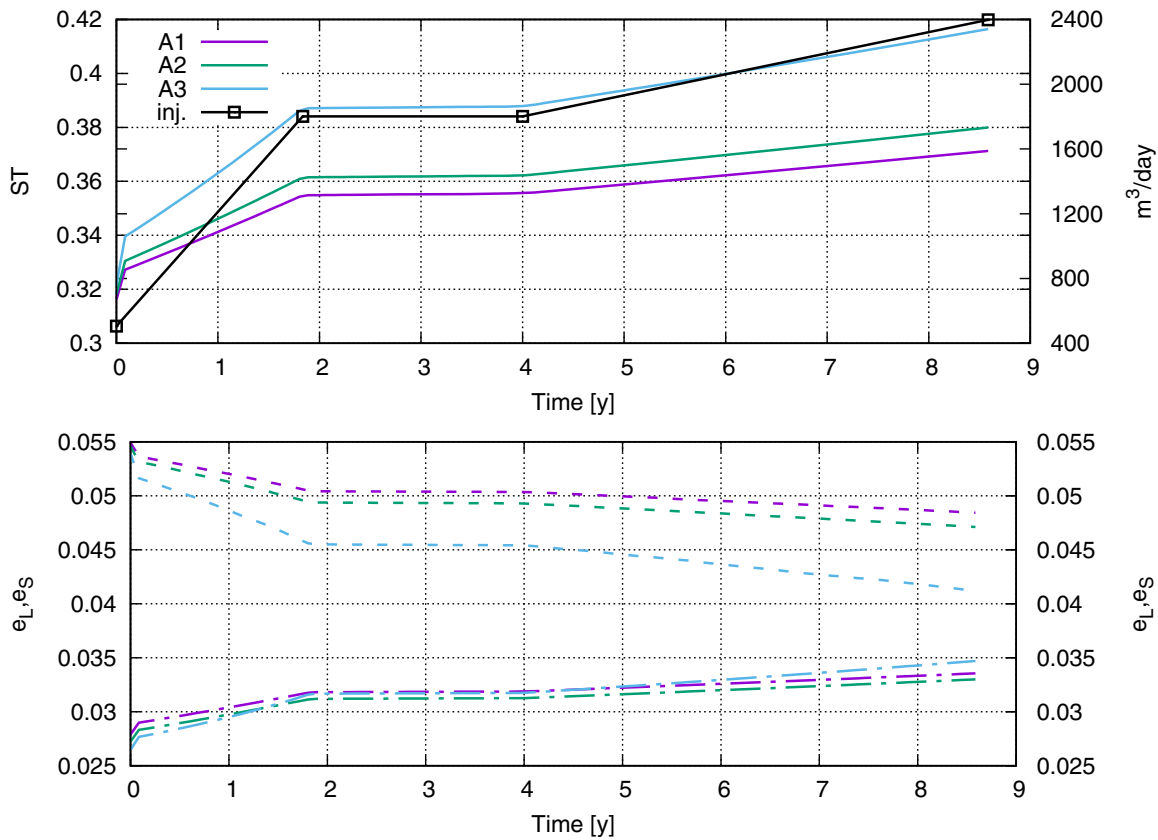

Fig. 3 Average ST (top) and ST standard deviation (bottom, divided in the contribution $e_{S}-$ with dashed lines, $e_{L}-$ with dotted-dashed lines) as function of time

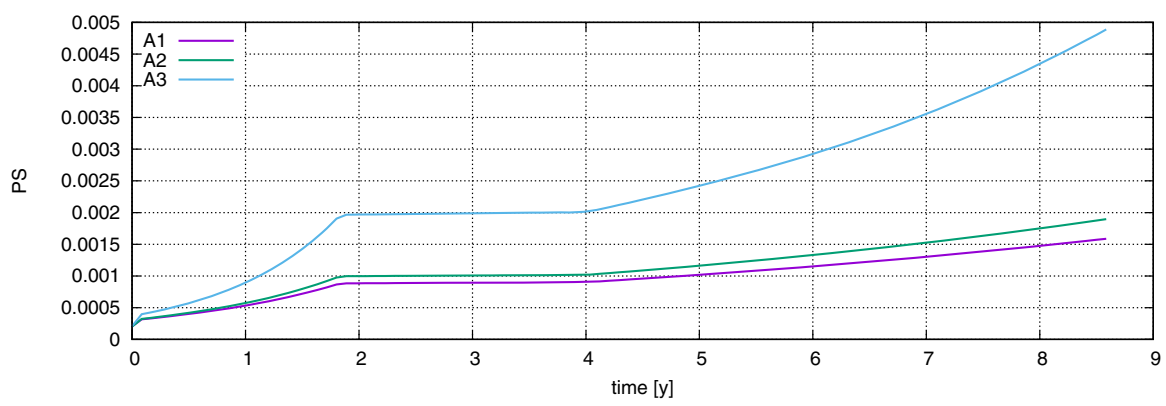

Fig. 4 History of the PS averaged over the fault elements, as a function of time

An effect of including into the analysis a probabilistic threshold on $\mu$ is visible in the spatially averaged PS values plotted versus time, as shown in Fig. 4. The graph for the 3 simulations follows closely the one for the average variances in Fig. 3, but it can be seen that to a linear increase in the injection rate corresponds a response in terms of PS that is more than linear. This can be ascribed to the different weighting of high and low values of ST. Another cause for this effect can be a non symmetrical form of the final distribution of ST, but this possibility has not yet been inspected.

The analysis of variances has been run on the last timestep only. For each element of the fault F1 we have computed the normal and a variation in azimuthal and zenithal 
Dip

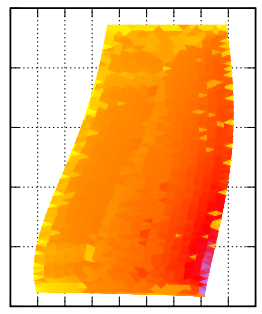

Dip+Perm

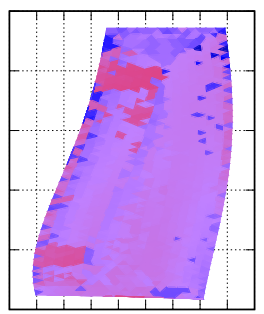

Strike

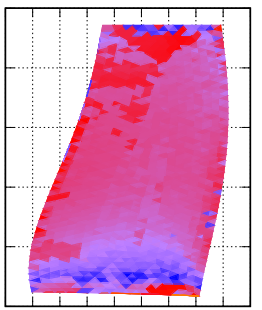

Dip+Strike

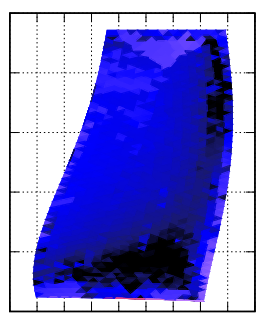

Perm.

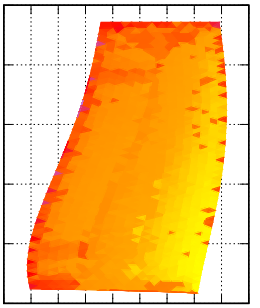

Strike+Perm.

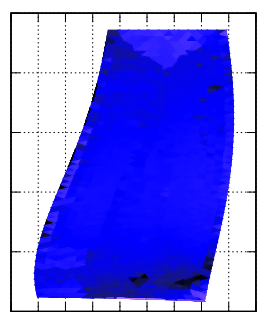

$1.00 \mathrm{e}+00$

$1.00 \mathrm{e}-01$

$1.00 \mathrm{e}-02$

$1.00 \mathrm{e}-03$

$1.00 \mathrm{e}-04$

$1.00 \mathrm{e}-05$

$1.00 \mathrm{e}-06$

$1.00 \mathrm{e}-07$

Residual

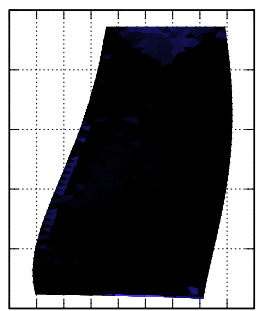

Fig. 5 Sensitivity analysis of the problem, with Dip, Strike and Permeability dependency

angle has been sampled at equally spaced intervals for a range $-5^{\circ},+5^{\circ}$. Each angle component has been sampled 100 times.

Results are shown in Fig. 5. About $99 \%$ of the variance is due to the 3 chosen parameters taken independently. Variations in the zenithal (dip) angle are the main source of variance in the top (left in the graph) border of the fault, and on the elements along the y boundary. The minor relevance of the azimuthal angle is expected since the largest of the principal stresses is oriented with the $z$ axis. In the remaining part of $\mathrm{F} 1$, variance is mostly associated with the variations of permeability. It is interesting to note that most of the microseismicity reported in Improta et al. (2015) is measured in the lower central-SE part (bottom right part of the map) of the fault, where the dependency of the variance of ST on the permeability is higher. The remaining sensitivity of combined variables covers for a small part of the total. The combined effect of permeability and dip variations is the most relevant and makes up for most of the remaining $1 \%$ of sensitivity. For the fault F1, then, there is no indication whether the source of variation can be ascribed more to the geometry of the fault or to the value of permeability, and the interplay between the two is large enough to become important when the ST is near its threshold $\mu_{\Gamma}$.

\section{Conclusions}

In this work, a statistical analysis including Uncertainty Quantification (UQ) and Sensitivity Analysis is applied to the relevant problem of fault stability. The tools are applied to a realistic case study with a coupled fluid flow and geomechanical model of fluid injection in the Val D'Agri oilfield with a time range of about 8.5 years. 
Simulations with three different settings for the damage zone permeability have been run, with the aim of testing how the fault stability is impacted.

The UQ algorithm inspects the stability of the fault through the Slip Tendency (ST) analysis and the evaluation of a new parameter, the Probability of Slip (PS), obtained by weighting the ST output with a statistical distribution depending on the average value and variance of the friction coefficient. Even if the value of PS and ST are small and far from critical, the method is able to show areas of higher instability which include the area where the microseismicity has been observed in reality. The analysis of variance on the permeability is able to discriminate, for values in the selected interval, where the permeability of the damage zone is dominating.

As a result of the UQ and sensitivity analysis, it is furthermore possible to state with a good amount of confidence that the mechanism causing the instability of the fault is still not evident from the model. This suggests future investigation with different initial/boundary stresses or including previously neglected effects, like a dependence of damage zone permeability on fluid pressure. To conclude, even with the evident limits deriving from its application as a post-processing tool, this statistical analysis can be used to drive the development of a dynamical model more compliant with reality.

Acknowledgements We would like to thank Mauro Buttinelli for the help with the interpretation of the fault geometries, Luca Formaggia and Stefano Micheletti for the useful discussions on the numerical issues about fault reactivation modelling and finally Luca Paglieri for the maintenance of the MOX-HPC facilities.

\section{References}

ABAQUS: Theory Manual, Version 6.13. Providence, Rhode Island (2013)

Aleksankina, K., Heal, M.R., Dore, A.J., Van Oijen, M., Reis, S.: Global sensitivity and uncertainty analysis of an atmospheric chemistry transport model: the frame model (version 9.15.0) as a case study. Geosci. Model Dev. 11, 1653-1664 (2018)

Bao, J., Chu, Y., Xu, Z., Tartakowsky, A.M., Fang, Y.: Uncertainty quantification for the impact of injection rate fluctuation on the geomechanical response of geological carbon sequestration. Int. J. Greenh. Gas Control 20, 160-167 (2014)

Buttinelli, M., Improta, L., Bagh, S., Chiarabba, C.: Inversion of inherited thrusts by wastewater injection induced seismicity at the val d'agri oilfield (italy). Sci. Rep. 6(37165), 1-8 (2016)

Collettini, C., Niemeijer, A., Viti, C., Marone, C.: Fault zone fabric and fault weakness. Nature 462, 907 (2009). EP

Colombo, I., Nobile, F., Porta, G., Scotti, A., Tamellini, T.: Uncertainty quantification of geochemical and mechanical compaction in layered sedimentary basins. Comput. Methods Appl. Mech. Eng. 328, 122-146 (2018)

Csanady, G.T.: Turbulent Diffusion in the Environment. D. Reidel Publishing, Dordrecht (1973)

Ellsworth, W.L.: Injection-induced earthquakes. Science 341, 6142 (2013). https://doi.org/10.1126/science. 1225942

Formaggia, L., Guadagnini, A., Imperiali, I., Lever, V., Porta, G., Riva, M., Scotti, A., Tamellini, L.: Global sensitivity analysis through polynomial chaos expansion of a basin-scale geochemical compaction model. Comput. Geosci. 17, 25-42 (2013)

Hall, J., Solomatine, D.: A framework for uncertainty analysis in flood risk management decisions. Int. J. River Basin Manag. 6(2), 85-98 (2008)

Improta, L., Valoroso, L., Piccinini, D., Chiarabba, C.: A detailed analysis of wastewater-induced seismicity in the val d'agri oil field (italy). Geophys. Res. Lett. 42, 2682-2690 (2015)

Jha, B., Juanes, R.: Coupled multiphase flow and poromechanics: a computational model of pore pressure effects on fault slip and earthquake triggering. Water Resour. Res. 50, 3776-3808 (2014) 
Kalra, T.S., Aretxabaleta, A., Seshadri, P., Ganju, N.K., Beudin, A.: Sensitivity analysis of a coupled hydrodynamic-vegetation model using the effectively subsampled quadratures method (esqm v5.2). Geosci. Model Dev. 10, 4511-4523 (2017)

Maina, F.Z., Guadagnini, A.: Uncertainty quantification and global sensitivity analysis of subsurface flow parameters to gravimetric variations during pumping tests in unconfined aquifers. Water Resour. Res 54, 501-518 (2018)

Moeck, I., Kwiatek, G., Zimmerman, G.: Slip tendency analysis, fault reactivation potential and induced seismicity in a deep geothermal reservoir. J. Struct. Geol. 31, 1174-1182 (2009)

Molini, L., Parodi, A., Siccardi, F.: Dealing with uncertainty: an analysis of the severe weather events over italy in 2006. Nat. Hazards Earth Syst. Sci. 9, 1775-1789 (2009)

Montone, P., Mariucci, M.T.: The new release of the italian contemporary stress map. Geophys. J. Int. 205(3), 1525-1531 (2016)

Morris, A.P., Ferrill, D.A.: The importance of the effective intermediate principal stress $\left(\sigma_{2}^{\prime}\right)$ to fault slip patterns. J. Struct. Geol. 31, 950-959 (2009)

Peng, P., Kumar, A., van den Dool, H., Barnston, A.G.: An analysis of multimodel ensemble predictions for seasonal climate anomalies. J. Geophys. Res. 107(D23), 1-12 (2002). https://doi.org/10.1029/ 2002JD002712

Pereira, L.C., Guimaraes, L.J.N., Horowitz, B., Sánchez, M.: Coupled hydro-mechanical fault reactivation analysis incorporating evidence theory for uncertainty quantification. Comput. Geotech. 56, 202-215 (2014)

Roy, P.T., Mocayd, N.E., Ricci, S., Goutal, N., De Lozzo, M., Rochoux, M.C.: Comparison of polynomial Chaos and Gaussian process surrogates for uncertainty quantification and correlation estimation of spatially distributed open-channel steady flows. Stoch. Envirn. Res. Risk Assess. 32, 1723-1741 (2018)

Saltelli, A., Tarantola, S.: On the relative importance of input factors on mathematical models: safety assesment for nuclear waste disposal. J. Am. Stat. Assoc. 97(459), 414-702 (2002)

Scuderi, M.M., Collettini, C.: The role of fluid pressure in induced vs. triggered seismicity: insights from rock deformation experiments on carbonates. Sci. Rep. 6, 24852 (2016)

Sibson, R.H.: A note on fault reactivation. J. Struct. Geol 7(6), 751-754 (1985). https://doi.org/10.1016/ 0191-8141(85)90150-6

Sobol, I.M.: Sensitivity estimates for nonlinear mathematical models. Comput. Math. Math. Phys. 1(4), 407-414 (1993)

Stabile, T.A., Giocoli, A., Perrone, A., Piscitelli, S., Lapenna, V.: Fluid injection induced seismicity reveals a ne dipping fault in the southeastern sector of the high agri valley (southern italy). Sci. Rep. 41, $5847-5854$ (2014)

Turcotte, D., Shubert, G.: Geodynamics. Cambridge University Press, Cambridge (2014)

Vadacca, L., Colciago, C.M., Micheletti, S., Scotti, A.: Effects of the anisotropy of the fault zone permeability on the timing of triggered earthquakes: insights from $3 \mathrm{~d}$-coupled fluid flow and geomechanical deformation modeling. Pure Appl. Gephys. 175, 4131-4144 (2018)

Vadacca, L., Rossi, D., Scotti, A., Buttinelli, M.: Effects of geological barriers on the induced seismicity by fluid injection: geomechanical analysis of a compartmentalized reservoir. In: Geophysical Research Abstracts, vol. 21, EGU2019-7387-1, EGU General Assembly (2019)

Vidal-Gilbert, S., Nauroy, J.F., Brosse, E.: 3d geomechanical modelling for co 2 geologic storage in the dogger carbonates of the paris basin. Int. J. Greenh. Gas Control 3, 288-299 (2009)

Wangen, M.: Physical Principles of Sedimentary Basin Analysis. Cambridge University Press, Cambridge (2019)

Worum, G., van Wees, J.D., Bada, G., van Balen, R., Cloetingh, S., Pagnier, H.: Slip tendency analysis as a tool to constrain fault reactivation: a numerical aproach applied to three-dimensional fault models in the roer valley rift system (southeast netherlands). J. Geophys. Res. 109(B02401), 1-16 (2004)

Publisher's Note Springer Nature remains neutral with regard to jurisdictional claims in published maps and institutional affiliations. 


\title{
Affiliations
}

\section{Daniele Rossi ${ }^{1}$ - Anna Scotti ${ }^{1}$. Luigi Vadacca ${ }^{1}$}

\author{
Anna Scotti \\ anna.scotti@polimi.it \\ Luigi Vadacca \\ luigi.vadacca@polimi.it \\ 1 Dip. Matematica, Politecnico di Milano, Via Bonardi 9, Milan, Italy
}

